\title{
Relationships among Three Multiplicities of a Differential Operator's Eigenvalue
}

\author{
Shouzhong Fu, Zhong Wang* \\ School of Mathematics and Statistics, Zhaoqing University, Guangdong, China \\ Email: szfu@zqu.edu.cn, ${ }^{*}$ kyczwang@zqu.edu.cn
}

Received 23 May 2014; revised 30 June 2014; accepted 14 July 2014

Copyright (C) 2014 by authors and Scientific Research Publishing Inc.

This work is licensed under the Creative Commons Attribution International License (CC BY). http://creativecommons.org/licenses/by/4.0/

c) (i) Open Access

\begin{abstract}
In this paper, the algebraic, geometric and analytic multiplicities of an eigenvalue for linear differential operators are defined and classified. The relationships among three multiplicities of an eigenvalue of the linear differential operator are given, and a fundamental fact that the algebraic, geometric and analytic multiplicities for any eigenvalue of self-adjoint differential operators are equal is proven.
\end{abstract}

\section{Keywords}

Differential Operators, Eigenvalue, Algebraic Multiplicities, Geometric Multiplicities, Analytic Multiplicities

\section{Introduction}

The study of spectral problems for linear ordinary differential equations (more generally, quasi-differential equations, to be abbreviated as QDE) originated from a series of seminal papers of Sturm and Liouville in [1]-[3], while the singular case started with the celebrated work of Weyl in 1910 introducing the limit-point (LP) and limit-circle (LC) dichotomy [4]. Another important milestone in this area is the Glazman-Krein-Naimark (GKN) theorem [5] in 1950, see also [6] for generalizations (which will be included in the theorem). This theorem gives a one-to-one correspondence between the self-adjoint differential operators in a Hilbert function space representing a given QDE and the unitary isometries on an appropriate finite-dimensional subspace (or equivalently, certain Lagrange subspaces of some finite dimensional quotient space, see [7]). In both regular case and singular case, the GKN theorem also yields a characterization of the self-adjoint operators in terms of linear complex boundary conditions (BC). So, the spectral problem of a linear ordinary differential equation (QDE) with boundary conditions maybe turn to study it of a linear ordinary differential operator [5] [8] [9]. It is well-known that

*Corresponding author. 
the investigation of different self-adjoint extensions of symmetric operators and the estimation of the location and multiplicity of their point spectra are among fundamental mathematical problems arising in any quantum mechanical model ([10], Chapter VIII, Section 11). While the eigenvalues of a linear ordinary differential operator were studied, three kinds of multiplicity (analytic multiplicity, geometric multiplicity and algebraic multiplicity) of an eigenvalue were defined and accompanied. Three kinds of multiplicity are often confused in some paper, and a problem how about the relationships among three kinds of multiplicity of an eigenvalue has arisen.

The differential equation

$$
I(y)=(-1)^{k}\left(p_{0}(x) y^{(k)}\right)^{(k)}+(-1)^{(k-1)}\left(p_{1}(x) y^{(k-1)}\right)^{(k-1)}+\cdots+p_{k}(x) y=\lambda w y, \quad x \in(a, b),
$$

with boundary conditions

$$
U_{j}(y)=0, \quad j=1,2, \cdots, 2 k
$$

will be studied in present paper, where $p_{0}(x)>0$ and $w(x)>0$ a.e. on $(a, b)$, the functions $1 / p_{0}(x), p_{1}(x), \cdots, p_{k}(x), w(x)$ are real-valued, measurable over $(a, b)$ and Lebesgue integrable on all compact subset of $(a, b)$.

The endpoint $a$ is said to be regular if $a>-\infty$ and each of the functions $1 / p_{0}(x), p_{1}(x), \cdots, p_{k}(x), w(x)$ is integrable in every interval $[a, c], c<b$; otherwise $a$ is said to be singular. Similar definitions apply to endpoint $b$. The differential expression $l(y)$ is said to be regular if it is regular at both endpoints, and otherwise is said to be singular.

We assume throughout that (1.1) is regular, and the functions $p_{j}(x)(0 \leq j \leq k)$ are sufficiently smooth and Lebesgue integrable on $(a, b)$, then the boundary conditions may be written as $(j=1,2, \cdots, 2 k)$ :

$$
U_{j}(y)=a_{j 1} y(a)+a_{j 2} y^{\prime}(a)+\cdots+a_{j k} y^{(2 k-1)}(a)+b_{j 1} y(b)+b_{j 2} y^{\prime}(b)+\cdots+b_{j k} y^{(2 k-1)}(b) .
$$

In the regular case, the GKN characterization of self-adjointness in terms of the complex boundary conditions can be simply expressed as the algebraic equation

$$
A Q^{-1}(a) A^{*}=B Q^{-1}(b) B^{*}
$$

and $\operatorname{Rank}(A \mid B)=2 k$, where $A=\left(a_{i j}\right)_{2 k \times 2 k}$ and $B=\left(b_{i j}\right)_{2 k \times 2 k}$ which come from the coefficient matrix $(A \mid B)$ of the boundary conditions are $2 k \times 2 k$ complex matrices, while $Q(t)$ is a fixed matrix for Lagrange bilinear form $[\cdot, \cdot]$ of differential expression $l(y)$ in (1.1).

Let $C(y(t))=\left(y(t), y^{\prime}(t), \cdots, y^{(n-1)}(t)\right)$, and $C^{t}(y(t))$ be the transpose of $C(y(t))$, then, for

$f, g \in D_{M}$ (the domain of maximal operator generated by $l(y)$ ),

$$
\begin{gathered}
(l(f), g)-(f, l(g))=[f, g]_{a}^{b}=[f, g](b)-[f, g](a), \\
{[f, g](x)=\overline{C(g(x))} Q(x) C^{t}(f(x)),}
\end{gathered}
$$

and following results are true (see [5] [6] [8]):

1) $Q^{*}(t)=-Q(t)$;

2) $\left(Q^{-1}(t)\right)^{*}=\left(Q^{*}(t)\right)^{-1}=-Q^{-1}(t)$.

It is well-known that the spectrum of such a problem consists of an infinite number of real eigenvalues and has no finite accumulation point. The eigenvalues are precisely the zeros of an entire function $\Delta(\lambda)$, called the characteristic function of the problem. The analytic multiplicity of an eigenvalue is the order of the eigenvalue as a zero of $\Delta(\lambda)$, the geometric multiplicity of an eigenvalue is the number of linearly independent eigenfunctions for the eigenvalue, and the algebraic multiplicity of an eigenvalue is the dimension of its root subspace (a subspace is spanned by the eigenvectors and its associated vectors).

The analytic multiplicity of an eigenvalue gives the maximum number of new eigenvalues into which the original eigenvalue can split when the spectral problem involved. So, it is natural to use the analytic multiplicity to count eigenvalues, and the analytic multiplicity plays an important role in the study of the dependence of the eigenvalues of a spectral problem on the differential equation boundary value problem (see, for example, [5] 
[11]-[14]). The geometric multiplicity is always defined and is more widely used in spectral theory (see [4] [5] [8]-[10] [15]). However, the algebraic multiplicities and associated vectors (functions) of an eigenvector (eigenfunction) are used for the completeness of eigenvectors (eigenfunctions) of non self-adjoint operators (see [15][17]). Therefore, it is of fundamental interest to compare the three multiplicities of an eigenvalue for differential operators.

Naimark studied the relationship between the algebraic and analytic multiplicities of an eigenvalue of highorder linear differential operators in [5], and obtained the equivalence of the algebraic and analytic multiplicities of an eigenvalue of high-order linear differential Equation (1.1) with linear boundary conditions (1.2). From then, the relationships among the three multiplicities have been payed a good deal of attentions, and have had a strong appeal to studying.

Over the last decades, the fact that the analytic and geometric multiplicities of an eigenvalue of self-adjoint Sturm-Liouville problems are equal has been solved ([5] [11]-[14] [18]-[22]). Sturm-Liouville problems (SLP) are differential equation:

$$
-\left(p y^{\prime}\right)^{\prime}+q y=\lambda w y \quad \text { on }(a, b)
$$

with boundary conditions

$$
A Y(a)+B Y(b)=0
$$

where $w>0$ a.e. on $(a, b), Y=\left(y, y^{\prime}\right)^{t}, A$ and $B$ are $2 \times 2$ matrices, $(A \mid B) \in M_{2,4}^{*}(C)$.

For the regular SLP, i.e. both endpoint $a$ and $b$ are regular $(-\infty<a<b<\infty, 1 / p, q, w \in L((a, b), R))$, the problem (1.4)-(1.5) is a self-adjiont SLP if the coefficients matrixes in (1.5) satisfy (1.3) and $\operatorname{Rank}(A \mid B)=2$.

The equality of the analytic and geometric multiplicities in the case of separated boundary conditions (BC) was proved in [13], while the case of coupled BC's was settled in [18]. The equality of the two multiplicities in the case of singular self-adjoint SLP with LC non-oscillatory end points was shown in [19] using a regularization; the equality in the case of all singular self-adjoint SLP with LC end points was recently established in [21], based on the equality of the regular self-adjoint SLP and certain regular approximations.

The proof in [18] uses some sophisticated identities involving $\Delta(\lambda)$ (the function $D(\lambda)$ in [18] differs from $-\Delta(\lambda)$ by a constant) and certain values of a fundamental set of solutions of (1.4). It seems to us that it is very hard to find similar identities for a higher order QDE by this way.

The basic idea of proof in [19] is as follows: for any eigenvalue $\lambda_{*}$ of geometric multiplicity 1 , they can give a smooth curve in the space $B^{C}$ of self-adjoint BC through the BC $\boldsymbol{A}$ involved such that the composition of $\Delta(\lambda)$ with a continuous eigenvalue branch through $\lambda_{*}$ has a non-zero derivative along the curve at $\boldsymbol{A}$, which then implies that $\Delta(\lambda)$ has a non-zero derivative at $\lambda_{*}$. Here, a continuous eigenvalue branch through $\lambda_{*}$ means a continuous function defined on a neighborhood $\boldsymbol{O}$ of $\boldsymbol{A}$ in $B^{C}$ such that its value at $\boldsymbol{A}$ equals $\lambda_{*}$ and its value at each BC $\boldsymbol{B} \in O$ is an eigenvalue for $\boldsymbol{B}$.

In [22], we generalized the proof in [19], and gave a new and unified proof of the equality between the analytic and geometric multiplicities of any eigenvalue of self-adjoint SLP in the regular case based on the geometric classifications of self-adjoint BC.

The classifications of self-adjoint BC about higher order differential operator are more complicated in geometric [7], and it seems rather complicated to find similar identities for a higher order QDE by the same method in [22]. Recently, this result was generalized to the higher order differential equations with self-adjoint boundary conditions in [23], but the proof was not easy.

In order to classify three multiplicities of an eigenvalue for linear differential operators, to obtain the relationships among three multiplicities, and to have a short and non-technical presentation so that the main idea of the general proof can be made transparent, we only give the general proof for regular self-adjoint QDE in this paper. For arbitrary self-adjoint $n$ th-order QDE in singular end points with defect index $n$, the proof is basically the same (with only obvious minor changes), but the introduction of the self-adjoint BC and the definition of the characteristic function are more involved (see, for example, [7] or [24]).

It is the main purpose, therefore, in the present work, to give the definitions of three kinds of multiplicities of an eigenvalue for linear differential operators and the relationships among them. In Section 2, we give the definitions of the geometric and algebraic multiplicities and the relationship between them. The definition of the 
analytic multiplicity for an eigenvalue of linear differential operators and the relationship between its analytic and algebraic multiplicities is given in Section 3. In last section, we have the equalities among three multiplicities of an eigenvalue for a self-adjoint linear differential operator.

\section{Geometric and Algebraic Multiplicities}

The definitions of the geometric and algebraic multiplicities for an eigenvalue of a linear operator are from [15]. Recall that a complex number $\lambda_{0}$ is called an eigenvalue of linear operator $T$ if there exists a non-zero element $y_{0} \in D(T)$ such that $T y_{0}=\lambda_{0} y_{0}$; in this case, $y_{0}$ is called an eigenfunction of $T$ for $\lambda_{0}$. The eigenfunctions for $\lambda_{0}$ span a subspace of $D(T), N_{\lambda_{0}}(T)$, called the eigenspace for $\lambda_{0}$; and the geometric multiplicity of $\lambda_{0}$ is the dimension of its eigenspace, denoted by $n_{\lambda_{0}}(T)$, i.e.

$$
n_{\lambda_{0}}(T)=\operatorname{dim} N_{\lambda_{0}}(T) \text {. }
$$

A non-zero element $y \in D(T)$ is called a root vector of $T$ for a complex number $\lambda_{0}$ if $\left(T-\lambda_{0} I\right)^{n} y=0$ for some $n \in N$. In this case, $\lambda_{0}$ must be an eigenvalue. Together with the vector $\phi=0$, the root vectors of $\lambda_{0}$ span a linear subspace of $D(T), M_{\lambda_{0}}(T)$, called the root lineal for $\lambda_{0}$; and the algebraic multiplicity of $\lambda_{0}$ is the dimension of its root lineal, denoted by $m_{\lambda_{0}}(T)$, i.e.

$$
m_{\lambda_{0}}(T)=\operatorname{dim} M_{\lambda_{0}}(T) .
$$

If an element $y \in D(T)$ is not an eigenvector for $\lambda_{0}$, then it is a root vector for $\lambda_{0}$ if and only if there is a $k \in N$ such that $y_{0}$ is an eigenvector for $\lambda_{0}$ provided $y_{k}=y$ and $y_{j-1}=T y_{j}-\lambda_{0} y_{j}$ for $j=k, \cdots, 1$. A root vector is called an associated vector (or adjoint vector) if it is not an eigenvector. The theory of associated functions (vectors) of differential operator was originated by Keldysh [16].

In general, the system of eigenvectors and associated vectors of $T$ is not complete in Hilbert space $H$. From the definition of the algebraic multiplicities and geometric multiplicity of an eigenvalue of linear operator $T$ in $H$, the eigenvectors of $\lambda_{0}$ belong to root lineal of $\lambda_{0}$ (i.e. $N_{\lambda_{0}}(T) \subset M_{\lambda_{0}}(T)$ ), so, we have the following result:

Theorem 2.1. The geometric multiplicity of any eigenvalue of linear operator $T$ in Hilbert space does not exceed its algebraic multiplicity. i.e.

$$
n_{\lambda_{0}}(T) \leq m_{\lambda_{0}}(T) .
$$

In general, the algebraic multiplicity is grater than the geometric multiplicity of an eigenvalue of operators. For example.

Example 2.2. We consider an operator $A$ in $\mathbb{R}^{2}$,

$$
A=\left(\begin{array}{cc}
0 & -1 \\
1 & 2
\end{array}\right),
$$

$\lambda_{1}=\lambda_{2}=1$ are eigenvalues of operator $A, y_{0}=(1,-1)^{\mathrm{T}}$ is an eigenvector for the eigenvalue $\lambda=1$, and the eigenspace $N_{\lambda}(A)=\operatorname{span}\left\{y_{0}\right\}$. So, the geometric multiplicity of the eigenvalue $\lambda=1$ is equal to 1, i.e. $n_{\lambda}(A)=1$. But, $(A-\lambda I) y_{=}=y_{0}$ have solutions $y_{1}, y_{2}$, where $y_{1}=(0,-1)^{\mathrm{T}}, y_{2}=(-1,0)^{\mathrm{T}}$, the associated vector of $y_{0}$ is $y_{1}=(0,-1)^{\mathrm{T}}$ or $y_{2}=(-1,0)^{\mathrm{T}}$, and the root lineal $M_{\lambda}(A)=\operatorname{span}\left\{y_{0}, y_{1}\right\}$, or $\operatorname{span}\left\{y_{0}, y_{2}\right\}$. Thus, the algebraic multiplicity of eigenvalue $\lambda=1$ is equal to 2, i.e. $m_{\lambda}(A)=2$.

Example 2.3. We consider the differential equation

$$
-y^{\prime \prime}(x)=\lambda y(x), x \in[0,1]
$$

with boundary conditions

$$
\left\{\begin{array}{l}
y(0)+3 y(1)=0 \\
y^{\prime}(0)+\cos ^{2} \sqrt{\lambda} y^{\prime}(1)=0
\end{array}\right.
$$

in Hilbert space $L^{2}[0,1]$. After simple calculation, $\lambda=\pi^{2}$ is an eigenvalue of boundary problem (1.3)-(1.4), $y=\sin \pi x$ is corresponding eigenvector, and the eigenspace of $\lambda=\pi^{2}$ is $N_{\lambda}=\operatorname{span}\{y\}$. So, the geometric multiplicity of eigenvalue $\lambda=\pi^{2}$ is equal to 1 , i.e. $n_{\lambda}=1$. But, $(L-\lambda I) y_{1}=y$ have solutions $y_{1}$, where 
$y_{1}=\frac{1}{2 \pi} x \cos \pi x-\frac{3}{4 \pi} \cos \pi x$, but $(L-\lambda I) y_{2}=y_{1}$ has not any solutions, the associated vector of $y$ is $y_{1}$ and the root lineal $M_{\lambda}=\operatorname{span}\left\{y, y_{1}\right\}$. Thus, the algebraic multiplicity of eigenvalue $\lambda=\pi^{2}$ is equal to 2, i.e. $m_{\lambda}=2$.

If $T$ is a spacial operator in Hilbert space $H$, then the geometric multiplicity of any eigenvalues of linear operator $T$ maybe equal to its algebraic multiplicity, such as:

Theorem 2.4. If $T$ is a self-adjoint operator in Hilbert space $H$, then the geometric multiplicity of any eigenvalues of linear operator $T$ is equal to its algebraic multiplicity.

Proof: We only need to prove that the eigenspace for an eigenvalue $\lambda$ of operator $T$ is equal to the root lineal of $\lambda$.

From the definitions of eigenspace and root lineal of the eigenvalue $\lambda$, we have

$$
N_{\lambda}(T) \subset M_{\lambda}(T),
$$

where $N_{\lambda}(T)$ and $M_{\lambda}(T)$ represent the eigenspace and the root lineal of $\lambda, \lambda$ is an (isolated) eigenvalue of $T$, i.e. $\lambda \in \sigma_{d}(T)$.

If $N_{\lambda}(T) \neq M_{\lambda}(T) \quad\left(N_{\lambda}(T) \subset M_{\lambda}(T)\right)$, then there exist a associated function $f_{1} \quad\left(\in M_{\lambda}(T), f_{1}(x) \neq 0\right)$ and an eigenfunction $f\left(\in N_{\lambda}(T), f(x) \neq 0\right)$, such that $T f=\lambda f$, and $T f_{1}-\lambda f_{1}=f$.

From the last equation, we have

$$
(f, f)=\left(T f_{1}, f\right)-\lambda\left(f_{1}, f\right) .
$$

By self-adjointness of operator $T$, the eigenvalue $\lambda$ is a real number, and $(T f, g)=(f, T g)$ for any $f, g \in D(T)$. Then,

$$
(f, f)=\left(f_{1}, T f\right)-\lambda\left(f_{1}, f\right)=0,
$$

and there is a contradictory to the fact $f(x) \neq 0$. Therefore,

$$
N_{\lambda}(T)=M_{\lambda}(T),
$$

and the proof is complete.

\section{Analytic and Algebraic Multiplicities}

We also introduce some notations here and review some basic facts about the problem of differential Equation (1.1) with boundary conditions (1.2). Let $y_{1}(x, \lambda), y_{2}(x, \lambda), \cdots, y_{n}(x, \lambda)$ be the fundamental solution of (1.1) satisfying

$$
y_{j}^{(v-1)}(a, \lambda)=\left\{\begin{array}{ll}
0, & j \neq v \\
1, & j=v
\end{array} \quad j, v=1,2, \cdots, n,\right.
$$

and $W(x, \lambda)=W\left(y_{1}, y_{2}, \cdots, y_{n}\right)(x, \lambda)$ denote the Wronskian of (1.1) with respect to $y_{1}(x, \lambda), y_{2}(x, \lambda), \cdots$, $y_{n}(x, \lambda)$, then $W(a, \lambda)=1$. The determinant of matrix $\left(U_{i}\left(y_{j}\right)\right)_{n \times n}$ is denoted by $\Delta(\lambda)$, i.e.

$$
\Delta(\lambda)=\left|\begin{array}{cccc}
U_{1}\left(y_{1}\right) & U_{1}\left(y_{2}\right) & \cdots & U_{1}\left(y_{n}\right) \\
U_{2}\left(y_{1}\right) & U_{2}\left(y_{2}\right) & \cdots & U_{2}\left(y_{n}\right) \\
\vdots & \vdots & \ddots & \vdots \\
U_{n}\left(y_{1}\right) & U_{n}\left(y_{2}\right) & \cdots & U_{n}\left(y_{n}\right)
\end{array}\right| .
$$

Theorem 3.1. The $\Delta(\lambda)$ is an entire function of $\lambda$, and a complex number $\lambda$ is an eigenvalue of the boundary value problem consisting of (1.1) and (1.2) if and only if

$$
\Delta(\lambda):=\operatorname{det}(A+B W(b, \lambda))=0 .
$$

Proof: Simply calculate or see [8] and [5]. 
The entire function $\Delta(\lambda)$, unique up to a non-zero constant multiple, is called the characteristic function for the boundary value problem consisting of (1.1) and (1.2). The analytic multiplicity of an isolated eigenvalue is the order of the eigenvalue as a zero of $\Delta(\lambda)$, denoted by $d_{\lambda_{0}}$.

From the definition of analytic multiplicity, only the eigenvalues of boundary value problems have analytic multiplicity. In general, the analytic multiplicity isn't equal to the other multiplicities for an eigenvalue of some boundary value problems.

Example 3.2. We still study the boundary value problem (2.3)-(2.4) in Example 2.3. The characteristic function $\Delta(\lambda)$ for the boundary value problem (2.3) and (2.4) can be easily obtained after calculating.

$$
\Delta(\lambda)=(1+\cos \sqrt{\lambda})^{3}
$$

$\lambda=\pi^{2}$ is a 3-order zero of the $\Delta(\lambda)$, so, the analytic multiplicity of the eigenvalue $\lambda=\pi^{2}$ is 3 .

With the results in Example 2.3, we have proven that the geometric multiplicity, algebraic multiplicity and analytic multiplicity of the eigenvalue $\lambda=\pi^{2}$ of boundary value problem (1.3)-(1.4) are 1, 2 and 3, and $n_{\lambda}<m_{\lambda}<d_{\lambda}$ for the eigenvalue $\lambda=\pi^{2}$ of boundary value problem (1.3)-(1.4).

But the linear differential Equation (1.1) with linear boundary conditions (1.2), Naimark had the following theorem in [5] (Chapter: $P_{18}$ ).

Theorem 3.3. The analytic multiplicity of any eigenvalues of the boundary value problem consisting of (1.1) and (1.2) is equal to its algebraic multiplicity. i.e.

$$
d_{\lambda_{0}}=m_{\lambda_{0}} .
$$

The algebraic multiplicity of an eigenvalue of self-adjoint SLP factually is the analytic multiplicity in [11][14], because the authors realized that the equivalence of the algebraic and analytic multiplicities for any eigenvalues of self-adjoint SLP is a foundational fact.

\section{Equalities among Three Multiplicities of an Eigenvalue of Self-Adjoint Differential Operators}

In this section, we first collect some basic statements about higher order differential operator (especially, the self-adjoint differential operator with high-order), and then prove the equalities among analytic, algebraic and geometric multiplicities.

For any $n, m \in \mathbb{N}$, we use $M_{n, m}(\mathbb{C})$ to denote the vector space of $n \times m$ matrices with complex entries and $M_{n, m}^{*}(\mathbb{C})$ its open subset consisting of the elements with the maximum rank $\min \{n, m\} \cdot M_{n, m}(\mathbb{R})$ and $M_{n, m}^{*}(\mathbb{R})$ are defined similarly. When a capital Latin or Greek letter stands for a matrix, the entries of the matrix will be denoted by the corresponding lower case letter with two indices. If $A \in M_{n, m}(\mathbb{C})$, then $A^{t}$ and $A^{*}$ are the transpose and the complex conjugate transpose of $A$, respectively. The general linear group $G L(n, \mathbb{C}):=M_{n, n}(\mathbb{C})$ is a complex Lie group under the matrix multiplication, while the special linear group $S L(n, \mathbb{C})$ consists of all elements of $G L(n, \mathbb{C})$ with determinant 1 and is a Lie subgroup of $G L(n, \mathbb{C})$. $G L(n, \mathbb{R})$ and $S L(n, \mathbb{R})$ are defined similarly. Let $J$ be an open interval, bounded or unbounded. Assume that $S$ is one of the spaces $\mathbb{C}^{n}, \mathbb{R}^{n}, M_{n, m}(\mathbb{C})$ and $M_{n, m}(\mathbb{R})$. We denote by $L(J, S)$ the space of Lebesgue integrable $S$-valued functions on $J$, and $A C_{l o c}(J, S)$ the space of $S$-valued functions which are absolutely continuous on all compact subintervals of $J$.

For the rest of this paper, we use $n(\in \mathbb{N})$ to denote a fixed number satisfying $n \geq 2$, and $J=(a, b)$ with $-\infty<a<b<+\infty$. When $n$ is even $(n=2 k)$ and $J$ is finite interval, there is a special case of the QDE (1.1). This special case was studied by Naimark [5] and Weidman [9].

For any $p_{0}(x)>0$ and $w(x)>0$ a.e. on $[a, b], 1 / p_{0}(x), p_{1}(x), \cdots, p_{k}(x), w(x)$ are integrable over $(a, b)$, and $p_{j}(x)$ are $k-j(0 \leq j \leq k)$ times continuous differentiable function on $(a, b), n=2 k$. We define 


$$
P=\left(\begin{array}{cccccccc}
0 & 1 & 0 & \cdots & \cdots & \cdots & \cdots & 0 \\
\vdots & \ddots & \ddots & \ddots & & & & \vdots \\
\vdots & & \ddots & 1 & \ddots & & & \vdots \\
\vdots & & & 0 & \frac{1}{p_{0}} & \ddots & & \vdots \\
\vdots & & \cdots & p_{1} & 0 & 1 & \ddots & \vdots \\
\vdots & \cdots & \cdots & \cdots & & \ddots & \ddots & 0 \\
0 & p_{k-1} & \cdots & & & & \ddots & 1 \\
p_{k} & 0 & \cdots & \cdots & \cdots & \cdots & \cdots & 0
\end{array}\right),
$$

then $P$ also belongs to $\mathrm{\complement}\left(J, M_{n, n}(\mathbb{R})\right)$. Let $Y(x)=\left(y(x), y^{[1]}, y^{[2]}, \cdots, y^{[n-1]}\right)^{t}$, the quasi-derivatives of $y$ associated with $P$ are given by

$$
\begin{gathered}
y^{[j]}=y^{(j)} \text { for } j=0,1, \cdots, k-1, \\
y^{[k]}=p_{0} y^{(k)}, \\
y^{[j]}=\left(y^{[j-1]}\right)^{\prime}-p_{j-k} y^{(n-j)} \text { for } j=k+1, \cdots, n,
\end{gathered}
$$

and

$$
W=\left(\begin{array}{ccccc}
0 & 0 & \cdots & \cdots & 0 \\
0 & \ddots & \cdots & \cdots & 0 \\
\vdots & & \ddots & \cdots & \vdots \\
\vdots & & & \ddots & \vdots \\
0 & 0 & \cdots & \cdots & w(x)
\end{array}\right),
$$

then, ordinary differential Equation (1.1) is equivalent quasi differential equation (QDE)

$$
Q y=i^{n} y^{[n]}=\lambda w y .
$$

Thus, $P$ and $W$ can be used as the coefficient matrix of (4.2), (4.2) is equivalent to its matrix form

$$
Y^{\prime}=(P-\lambda W) Y \text { on } I \text {. }
$$

The quasi-differential expression in $y$ associated with $Q$ for $n=2$ or $n=4$ or $n=6$ are of special interest:

$$
\begin{gathered}
Q y=-\left(p_{0} y^{\prime}\right)^{\prime}+p_{1} y, \quad \text { if } n=2, \\
Q y=\left[\left(f_{0} y^{\prime \prime}\right)^{\prime}-p_{1} y^{\prime}\right]^{\prime}-p_{2} y, \quad \text { if } n=4, \\
Q y=\left\{\left[-\left(p_{0} y^{\prime \prime \prime}\right)^{\prime}+p_{1} y^{\prime \prime}\right]^{\prime}+p_{2} y^{\prime}\right\}^{\prime}+p_{3} y, \text { if } n=6,
\end{gathered}
$$

while, in general, $Q y$ is equal to

$$
(-1)^{k}\left\{\left\{\ldots\left\{\left[\left(p_{0} y^{(k)}\right)^{\prime}-p_{1} y^{(k-1)}\right]^{\prime}-p_{2} y^{(k-2)}\right\}^{\prime}-\cdots-p_{k-1} y^{\prime}\right\}^{\prime}-p_{k} y\right\} .
$$

We now turn to the BVP consisting of the general QDE (1.2) and a (linear two-point) BC defined by 


$$
C Y(a)+D Y(b)=0,
$$

where $C, D \in M_{n, n}(\mathbb{C})$ such that $(C \mid D) \in M_{n, 2 n}^{*}(\mathbb{C})$. Note that equivalent linear algebraic equations of the form (1.2) define the same BC. Each value of $\lambda$ for which the QDE (4.2) has a nontrivial solution satisfying the BC (1.4) is called an eigenvalue of the BVP consisting of (4.2) and (4.4), and a solution to this problem is called an eigenfunction for this eigenvalue.

Since (4.2) has exactly $n$ linearly independent solutions, the geometric multiplicity of any eigenvalue is an integer not smaller than 1 and not greater than $n$.

Theorem 4.1. A number $\lambda \in \mathbb{C}$ is an eigenvalue of the boundary value problem consisting of (4.2) and (4.4) if and only if

$$
D(\lambda):=\operatorname{det}(C+D \Phi(b, \lambda))=0 .
$$

where $\Phi(x, \lambda)$ is the fundamental solution of (1.3) satisfying $\Phi(a, \cdot)=I, \quad I \quad$ is the identity matrix.

Proof: Simply calculate or see [7]. $\square$

Theorem 4.2. $D(\lambda)=c \Delta(\lambda)$.

Proof: From the definition of quasi-derives of $y$ associated with $P$, we have $Y(x)=M(x) C^{t}(y(x))$, where $Y(x)=\left(y(x), y^{[1]}, y^{[2]}, \cdots, y^{[n-1]}\right)^{t}, \quad C^{t}(y(x))=\left(y(x), y^{\prime}, y^{(2)}, \cdots, y^{(n-1)}\right)^{t}$, and

$$
M(x)=\left(\begin{array}{ccccccccc}
1 & 0 & 0 & \ldots & \ldots & \ldots & \ldots & 0 & 0 \\
0 & 1 & 0 & \ldots & \cdots & \ldots & \ldots & 0 & 0 \\
\vdots & \ddots & \ddots & \ddots & & & & \vdots & \vdots \\
\vdots & & \ddots & 1 & \ddots & & & \vdots & \vdots \\
\vdots & & & 0 & p_{0}(x) & \ddots & & \vdots & \vdots \\
\vdots & & \ldots & -p_{1}(x) & p_{0}^{\prime}(x) & p_{0}(x) & \ddots & \vdots & \vdots \\
\vdots & \ldots & \ldots & \ldots & & \ddots & \ddots & 0 & 0 \\
0 & p_{k-1} & \ldots & & & & \ddots & p_{0}(x) & 0 \\
p_{k} & * & \ldots & \ldots & \ldots & \ldots & \ldots & * & p_{0}(x)
\end{array}\right),
$$

is a inverse matrix function on $[a, b]$ because of $p_{0}(x)>0$ over $[a, b]$. Let $W(x, \lambda)$ denote the Wronskian of (1.1) with respect to $y_{1}(x, \lambda), y_{2}(x, \lambda), \cdots, y_{n}(x, \lambda), \Phi(x, \lambda)=M(x) W(x, \lambda) M^{-1}(a)$, then $A=C M(a), B=D M(b)$, and $\Phi(a, \lambda)=I$. Thus, $D(\lambda)=\Delta(\lambda) \cdot \operatorname{det} P^{-1}(a)$ and $c=\operatorname{det} P^{-1}(a)$. So, the equality in this theorem was proved.

A coefficient matrix $F$ is said to be symmetric if

$$
F E+E F^{*}=0,
$$

where

$$
E=\left(\begin{array}{cccc}
0 & \cdots & 0 & -1 \\
\vdots & \cdots & (-1)^{2} & 0 \\
0 & \cdots & \ddots & \vdots \\
(-1)^{n} & 0 & \cdots & 0
\end{array}\right) .
$$

The boundary condition (1.4) is said to be self-adjoint if $\operatorname{Rank}(C \mid D)=2 k$ and

$$
C E C^{*}=D E D^{*} \text {. }
$$

Theorem 4.3. The differential operator $L$ associated with (1.1) and (1.2) (or (4.2) and (4.4)) is a self-adjoint operator if the boundary conditions (1.2) (or (4.4)) satisfy (1.3) and $\operatorname{Rank}(A \mid B)=2 k$ (or is self-adjoint).

Proof: See [7]. $\square$

Theorem 4.4. The analytic, algebraic and geometric multiplicity of any eigenvalue for a self-adjoint differential operator associated with (1.1) and (1.2) (or (4.2) and (4.4)) are equal. 
Proof: From Theorem 2.4 and Theorem 3.3, we get the conclusion immediately. $\square$

Corollary 4.5. The analytic, algebraic and geometric multiplicity of any eigenvalue of the differential operator $L$ associated with (1.1) and (1.2) (or (4.2) and (4.4)) are equality when the coefficient matrixes $A$ and $B$ satisfy (1.3) (or $C$ and $D$ satisfy (4.9)), and equal to the multiplicities of the eigenvalue as a zero point of $\Delta(\lambda)$ in (3.3) (or $D(\lambda)$ in (4.5)).

Example 4.6. We consider the differential equation

$$
-y^{\prime \prime}(x)=\lambda y(x), x \in[0,1]
$$

with boundary conditions

$$
\left\{\begin{array}{l}
y(0)-y(1)=0 \\
y^{\prime}(0)-y^{\prime}(1)=0
\end{array}\right.
$$

in Hilbert space $L^{2}[0,1]$. After simple calculation, $\lambda=4 n^{2} \pi^{2},(n=0,1,2, \cdots)$ are eigenvalues of boundary problem (4.10)-(4.11), $y_{1}=\sin 2 n \pi x$ and $y_{2}=\cos 2 n \pi x$ are corresponding eigenvectors for $\lambda=4 n^{2} \pi^{2}$, and the eigenspace of $\lambda=4 n^{2} \pi^{2}$ is $N_{\lambda}=\operatorname{span}\left\{y_{1}, y_{2}\right\}$. So, the geometric multiplicity of eigenvalue $\lambda=4 n^{2} \pi^{2}$ is equal to 2, i.e. $n_{\lambda}=2$. Boundary problem (4.10)-(4.11) is a self-adjoint problem because of boundary conditions (4.11) satisfying (4.9), so, the algebraic multiplicity of eigenvalue $\lambda=4 n^{2} \pi^{2}$ is also equal to 2 , i.e. $m_{\lambda}=2$. We also have that

$$
\Delta(\lambda)=2 \sqrt{\lambda}(1-\cos \sqrt{\lambda})
$$

$\lambda=4 n^{2} \pi^{2}(n=1,2, \cdots)$ are 2-order zero of the $\Delta(\lambda)$, so, the analytic multiplicity of the eigenvalue $\lambda=4 n^{2} \pi^{2}$ is 2, i.e. $d_{\lambda}=2$. Thus, the analytic, algebraic and geometric multiplicity of any eigenvalue $\lambda=4 n^{2} \pi^{2}$ for boundary problem (4.10)-(4.11) are equal to 2, i.e. $d_{\lambda}=m_{\lambda}=n_{\lambda}=2$.

\section{Funding}

Work partially supported by the National Nature Science Foundation (11171295).

\section{References}

[1] Liouville, J. and Sturm, J.C.F. (1837) Extrait d'une méemoire sur le développement des fonctions en serie. Journal de Mathématiques Pures et Appliquées, 2, 220-223.

[2] Sturm, J.C.F. (1836) Mémoire sur les équations différentielles linéaires du second ordre. Journal de Mathématiques Pures et Appliquées, 1, 106-186.

[3] Sturm, J.C.F. (1837) Mémoire sur une classe d'équations différentielles partielles. Journal de Mathématiques Pures et Appliquées, 2, 373-444.

[4] Weyl, H. (1910) Üeber gewöhnliche differentialgleichungen mit singularitäten und die zugehörigen entwichlungen willkürlicher funktionen. Mathematische Annalen, 68, 220-269. http://dx.doi.org/10.1007/BF01474161

[5] Naimark, M.A. (1968) Linear Differential Operators. Ungar, New York.

[6] Everitt, W.N. and Markus, L. (1997) The Glazman-Krein-Naimark Theorem for Ordinary Differential Operators, New Results in Operator Theory and Its Applications. Operator Theory: Advances and Applications, 98, 118-130.

[7] Cao, X. and Wu, H. (2004) Geomtric Aspects of High-Order Eigenvalue Problems I. Structures on Spaces of Boundary Conditions. International Journal of Mathematics and Mathematical Sciences, 13, 647-678. http://dx.doi.org/10.1155/S0161171204303522

[8] Coddington, E. and Levinson, N. (1955) Theory of Ordinary Differential Equations. McGraw-Hill, New York.

[9] Weidmann, J. (1987) Spectral Theory of Ordinary Differential Operator, Lecture Notes in Mathematics, Vl 1258. Springer-Verlag, Berlin.

[10] Reed, M. and Simon, B. (1972) Methods of Modern Mathematical Physics I. Functional Analysis. Academic Press, Waltham.

[11] Kong, Q., Wu, H. and Zettl, A. (1997) Dependence of Eigenvalues on the Problem. Mathematische Nachrichten, 188, 173-201. http://dx.doi.org/10.1002/mana.19971880111

[12] Kong, Q., Wu, H. and Zettl, A. (1999) Dependence of the n-th Sturm-Liouville Eigenvalue on the Problem. Journal of Differential Equations, 156, 328-354. http://dx.doi.org/10.1006/jdeq.1998.3613 
[13] Kong, Q., Wu, H. and Zettl, A. (2000) Geometric Aspects of Sturm-Liouville Problems, I. Structures on Spaces of Boundary Conditions. Proceedings of the Royal Society of Edinburgh Section A, 130, 561-589.

[14] Zettl, A. (2005) Sturm-Liouville Theory. Mathematical Surveys and Monographs, Volume 121. American Mathematical Society.

[15] Gohberg, I.C. and Krein, M.G. (1969) Introduction to the Theory of Linear Non-Self-Adjoint Operator. Translation of Mathematical Monographs 18, American Mathematical Society, Providence.

[16] Keldysh, M.V. (1951) On Eigenvalues and Eigenfunctions of Some Classes of Non Self-Adjoint Equations. Doklady Akademii Nauk SSSR, 77, 11-14.

[17] Marcenko, V.A. (1963) Expansion in Eigenfuctions of Non-Self-Adjoint Singular Differential Operators of Second Order. American Mathematical Society Translations, 25, 77-130.

[18] Eastham, M., Kong, Q., Wu, H. and Zettl, A. (1999) Inequalities among Eigenvalues of Sturm-Liouville Problems. Journal of Inequalities and Applications, 3, 25-43.

[19] Kong, Q., Wu, H. and Zettl, A. (1999) Inequalities among Eigenvalues of Singular Sturm-Liouville Problems. Dynamic Systems and Applications, 8, 517-531.

[20] Kong, Q., Wu, H. and Zettl, A. (2001) Sturm-Liouville Problems with Finite Spectrum. Journal of Mathematical Analysis and Applications, 263, 748-762. http://dx.doi.org/10.1006/jmaa.2001.7661

[21] Kong, Q., Wu, H. and Zettl, A. (2004) Multiplicity of Sturm-Liouville Eigenvalues. Journal of Computational and Applied Mathematics, 171, 291-309. http://dx.doi.org/10.1016/j.cam.2004.01.036

[22] Wang, Z. and Wu, H. (2005) Equality of Multiplicities of a Sturm-Liouville Eigenvalue. Journal of Mathematical Analysis and Applications, 306, 540-547. http://dx.doi.org/10.1016/j.jmaa.2004.10.041

[23] Shi, D. and Huang, Z. (2010) Relationship of Multiplicities of a High-Order Ordinary Differential Operator Eigenvalue. Acta Mathematica Sinica, Chinese Series, 53, 763-772.

[24] Wang, Z. and Wu, H. (2009) Sturm-Liouville Problems with Limit-Circle End Points. Pacific Journal of Applied Mathematics, 1, 421-447. 
Scientific Research Publishing (SCIRP) is one of the largest Open Access journal publishers. It is currently publishing more than 200 open access, online, peer-reviewed journals covering a wide range of academic disciplines. SCIRP serves the worldwide academic communities and contributes to the progress and application of science with its publication.

Other selected journals from SCIRP are listed as below. Submit your manuscript to us via either submit@scirp.org or Online Submission Portal.
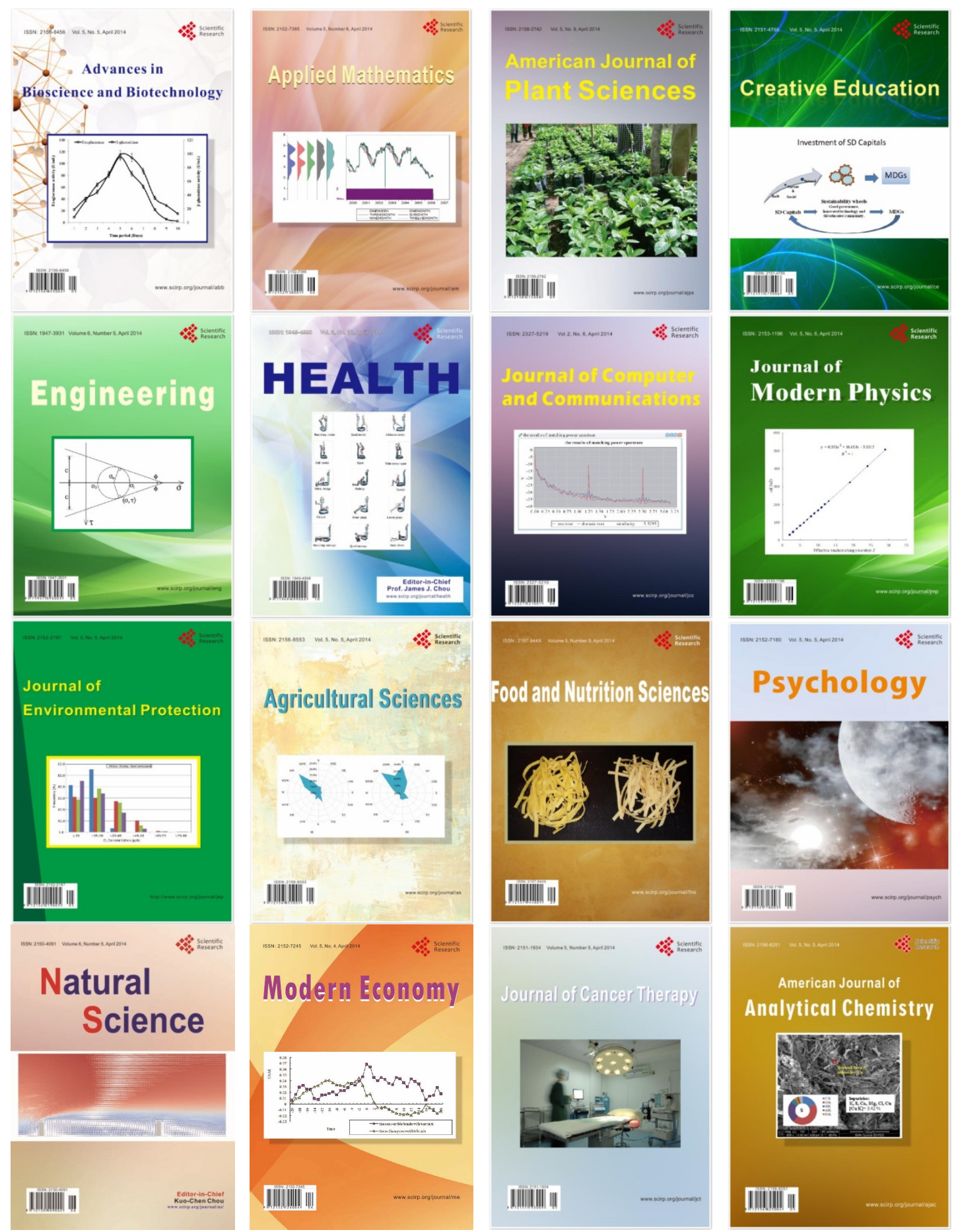